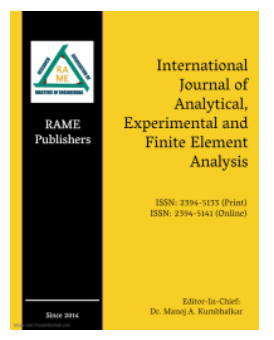

E. El Kennassi ${ }^{1}$ essaid.elkennassi@ieee.org

F. El Kennassi' ${ }^{2,3}$ elkennassi@gmail.com

M. A. Dirhar dirhar.medamine@gmail.com

E. Azelmad essaadia.ensem@gmail.com

K. I. Janati ${ }^{4}$

Janati.id02@gmail.com

${ }^{1}$ Mechanics Department, ENSEM, Hassan II University of Casablanca, Morocco.

${ }^{2}$ Cardiovascular Surgeon Moulay Youssef Hospital, Rabat, Morocco.

${ }^{3}$ Pharmacology and Toxicology Department, Faculty of medicine, Mohammed V University of Rabat, Morocco.

${ }^{4}$ Faculty of Sciences and Technologies, Sidi Mohamed Ben Abdellah University of Fez, Morocco.

\section{INTRODUCTION}

Researches on the mechanical properties of nano particles have attracted increasing interest especially for the biological cell. Our existence depends on cell functions instead of biochemical mechanisms, cell rely on mechanical behavior which is also related to extracellular matrix ECM. Research Article

Published online - 20 Aug 2020

(C) 2020 RAME Publishers

This is an open access article under the CC BY 4.0 International License https://creativecommons.org/licenses/by/4.0/

Cite this article - E. El Kennassi, F. El Kennassi, M. A. Dirhar, E. Azelmad, K. I. Janati, "Nano-Mechanical Eukaryotic Cell Behavior by Finite Element Modeling, International Journal of Analytical, Experimental and Finite Element Analysis, RAME Publishers, vol. 7, issue 3, pp. 61-67, 2020. https://doi.org/10.26706/ijaefea.3.7.20200603 element method.

\title{
Nano-Mechanical Eukaryotic Cell Behavior by Finite Element Modeling
}

Abstract-The cell mechanics behavior must be understood by the scientific community. There is two used methods: nanoindentation and atomic force microscopy AFM. The first one gives displacement between $10^{-9}$ and $10^{-3}$ meter corresponding to a load from $10^{-7}$ to 10 Newton. The second one gives displacement between $10^{-11}$ and $10^{-7}$ meter corresponding to a load ranging from $10^{-12}$ to $10^{-5}$ Newton. This work gives the nanoindentation eukaryotic cell simulation by the use of the commercial software: COMSOL Multiphysics and we give the relation to AFM. The nano-mechanical cell behavior was investigated using the finite element method, especially, we implement on it, the mechanics continuum. First, we created the 2D cell model. This model was constrained vertically at the bottom. We used hyperelastic model for the cytoplasm. Nanoindenter and cell contact was assumed to be a source boundary. In the second part of this work, we incorporated a circular section to the model. This circular section represents the nucleus chosen to be elastic. We then show that nucleus influences the cell mechanical response. After modeling and simulation, we obtain results in good agreements with those obtained experimentally.

Keywords - Multiphysics, eukaryotic cell, nanoindentation, modeling, COMSOL, finite

The mechanical cell behavior gives more details to understand disease and cancer properties [22,27]. Methods used to determine cell mechanical characteristics are AFM which gives the surface soft topology[10-11,14,19,20,22,2526,30-31,33], and nanoindentation [2,4-5,12,27-28,32]. This later is used to give mechanical cell behavior in experimental try with a rigid indenter and a rigid support. In this work we will model and simulate nanoindentation cell response to predict best conditions which can be used by experimental researcher [23,29,33,34]. First, we will model nanoindentation for a cell without nucleus and then we will give nanoindentation for eukaryotic cell with nucleus as shown in figure 1 and figure 2. 
Cells present an hyperelastic behavior [15-16,35] due to cytoskeletal tensegrity filaments [1,3,6-7]. The model used in this work is the hyperelasticity Neo-Hookean model $[15,17,35]$.

Our model was created by the contact finite element method $[9,21,24]$, and the results are simulated by the commercial software COMSOL Multiphysics [13,18,35].

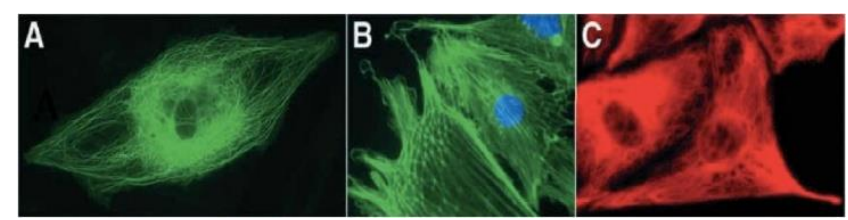

Figure 1. Endothelial cells cytoskeleton. A. microtubules.

B.Microfilaments. C. Intermediate filaments [7]

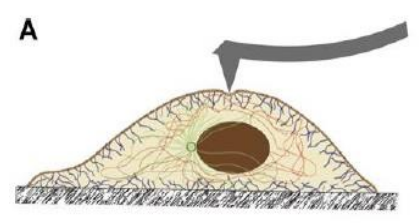

B

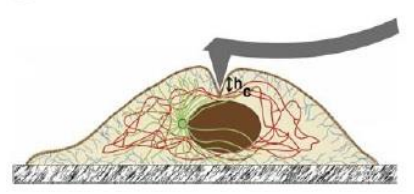

C

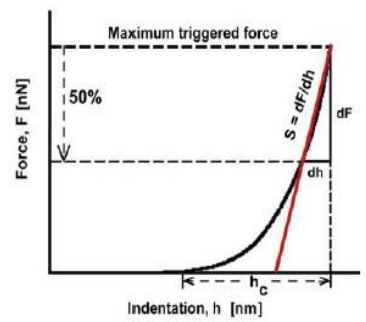

Figure 2. Model of the AFM indentation regime for analyzing Indentation Force nanomechanics in cells. A. AFM tip first encounters the actin cytoskeleton (blue). B. intermediate filament network (red). C. indentation hardness vs force curve [22].

Obtained results are in good agreements with those obtained experimentally [5,22].

TABLE I

Cell Material Parameters [8]

\begin{tabular}{|c|c|c|}
\hline Name & Value & Unit \\
\hline Density & $1200 \mathrm{~kg} / \mathrm{m}^{3}$ & $\mathrm{~kg} / \mathrm{m}^{3}$ \\
\hline Lamé parameter $\lambda$ & $144 \mathrm{e} 6 \mathrm{~N} / \mathrm{m}^{2}$ & $\mathrm{~N} / \mathrm{m}^{2}$ \\
\hline Lamé parameter $\mu$ & $7.2 \mathrm{e} 6 \mathrm{~N} / \mathrm{m}^{2}$ & $\mathrm{~N} / \mathrm{m}^{2}$ \\
\hline
\end{tabular}

TABLE II

NANOINDENTER AND RIGID SUPPORT MATERIAL PARAMETERS

\begin{tabular}{|c|c|c|}
\hline Name & Value & Unit \\
\hline Density & $3515 \mathrm{~kg} / \mathrm{m}^{3}$ & $\mathrm{~kg} / \mathrm{m}^{3}$ \\
\hline
\end{tabular}

\section{NUMERICAL EXPERIMENT}

The numerical experiment is based on the supposition that system model can be simulated assuming some assumption depending on the studied region. This later is formed by indenter, cell and a support as shown in figure 3 and figure 5.

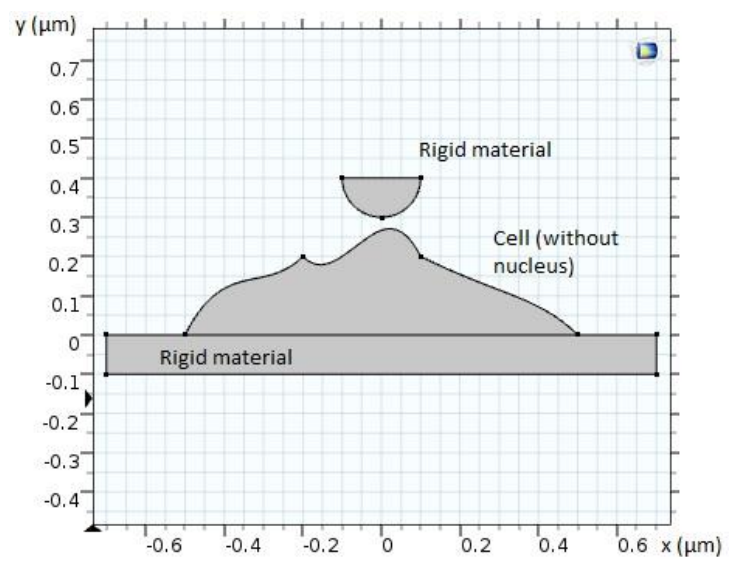

Figure 3: Cell without nucleus and indenter model geometry plus a rigid support designed with Comsol.

$$
\text { TABLE III }
$$

MESh GENERATION DETAILS

\begin{tabular}{|l|c|}
\hline Triangular elements & 280 \\
\hline Edge elements & 88 \\
\hline Vertex elements & 11 \\
\hline Mesh quality & 0.7 \\
\hline
\end{tabular}

\section{A. Nonlinear Structure Material}

Elastic Model: For the indenter and rigid support, we used equations for elastic model,

$$
\begin{aligned}
& -\nabla \sigma=F v \\
& \sigma=(S \cdot(I+\nabla u)) \\
& S-S_{0}=C:\left(\varepsilon-\alpha\left(T-T_{r e f}\right)-\varepsilon_{0}\right) \\
& \varepsilon=\frac{1}{2}\left[(\nabla u)^{T}+\nabla u+(\nabla u)^{T} \nabla u\right]
\end{aligned}
$$

where,

$\mathrm{S}$ is the second Pikola-Kirchhoff stress,

$\nabla u$ is the displacement gradient,

$F$ is the deformation gradient,

$v$ is the left stretch tensor, 
$\sigma$ is the Cauchy stress,

$\mathrm{C}$ is the right Cauchy-Green deformation tensor, $\varepsilon$ is the Green strain,

$\varepsilon_{0}$ is the initial strain,

$I$ is the identity tensor,

$T$ is present temperature,

$T_{r e f}$ is the stress free reference temperature,

$\alpha$ is the thermal expansions vector.

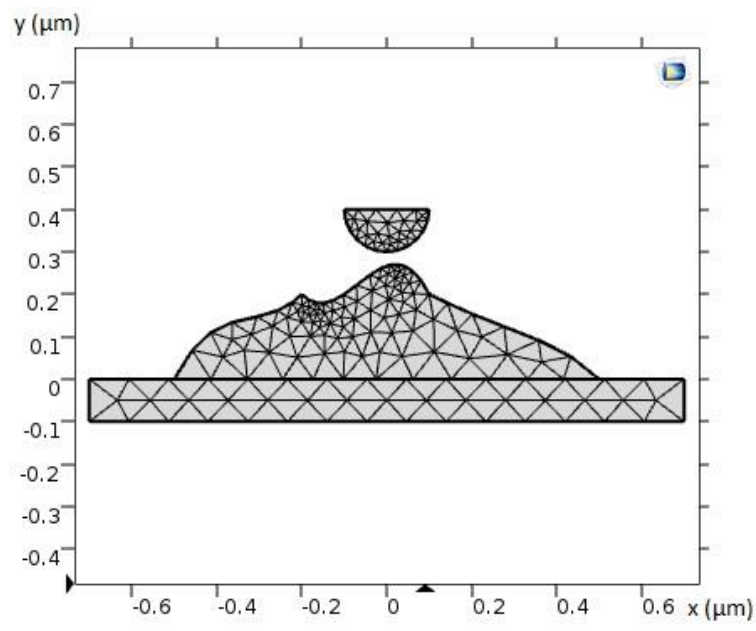

Figure 4: Obtained mesh generation.

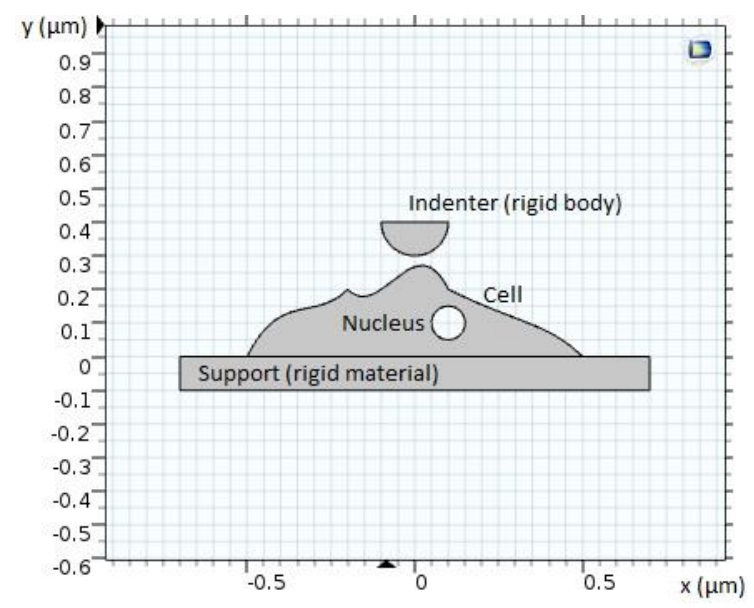

Figure 5: Cell with nucleus and indenter model geometry plus rigid support designed with comsol.

TABLE IV

Mesh GenaRATION DETAILS

\begin{tabular}{|l|c|}
\hline Triangular elements & 557 \\
\hline Edge elements & 125 \\
\hline Vertex elements & 15 \\
\hline Mesh quality & 0.8 \\
\hline
\end{tabular}
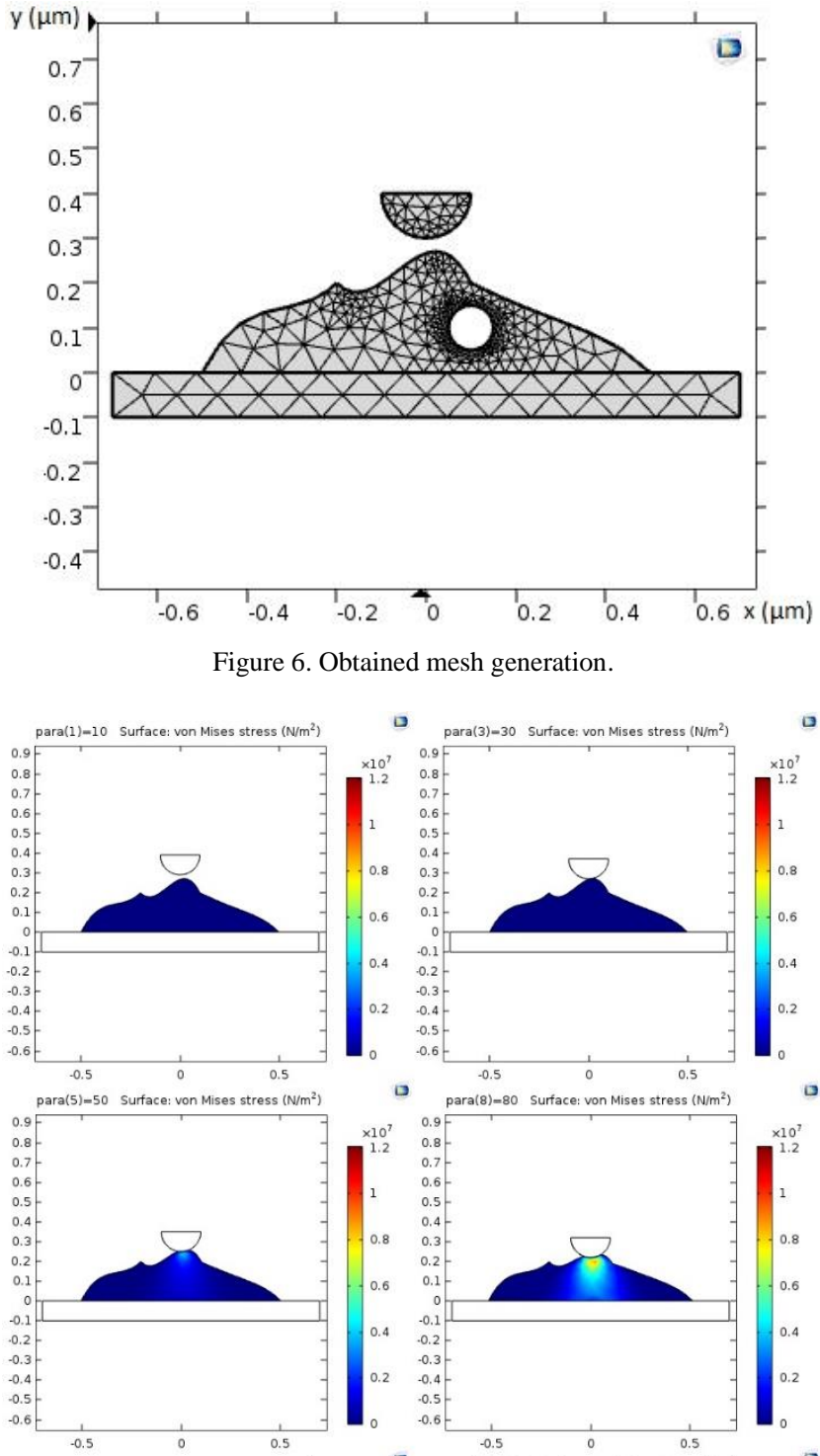

para(9) $=90$ Surface: von Mises stress $\left(\mathrm{N} / \mathrm{m}^{2}\right)$

$\square$
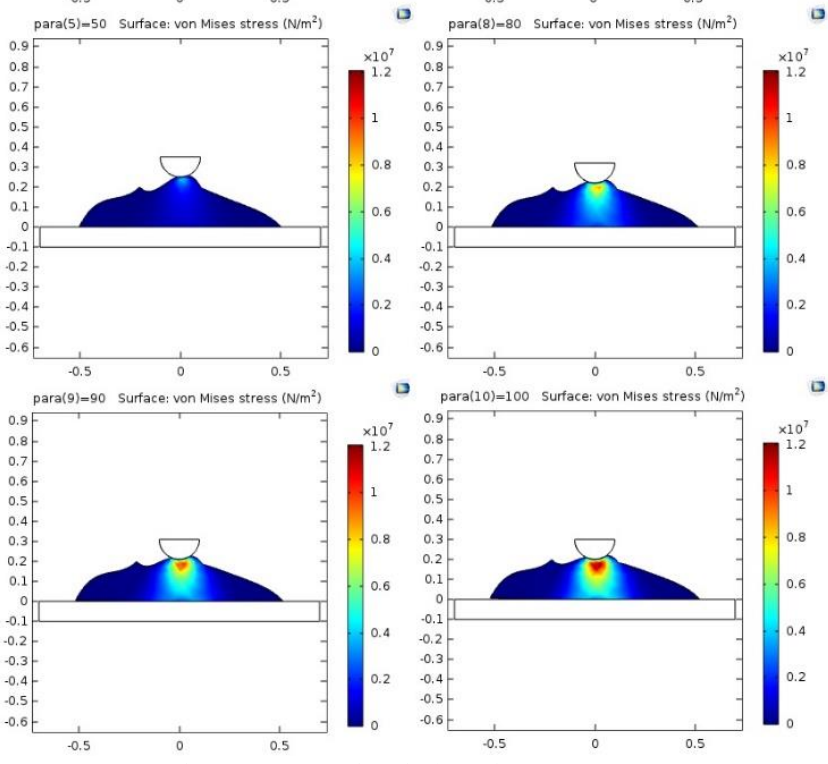

para(10) $=100$ surface: von Mises stress (N/mi

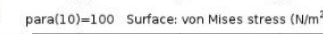

○

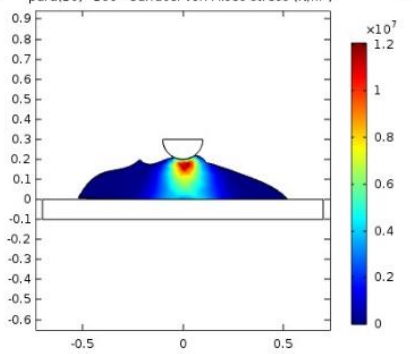

Figure 7: Von Mises indentation steps N/m².

Hyperelastic model (Neo-Hookean) - For the cell model, we use the Neo-Hookean hyperelastic model,

$$
\begin{aligned}
& -\nabla \sigma=F v \\
& \sigma=(S \cdot(I+\nabla u)) \\
& S=\frac{\partial W_{s}}{\partial \varepsilon}
\end{aligned}
$$




$$
\begin{aligned}
& W_{s}=\frac{1}{2} \mu\left(\bar{I}_{1}-3\right)-\mu \ln \left(J_{e l}\right)+\frac{1}{2} \lambda\left[\ln \left(J_{e l}\right)\right]^{2} \\
& \varepsilon=\frac{1}{2}\left[(\nabla u)^{T}+\nabla u+(\nabla u)^{T} \nabla u\right]
\end{aligned}
$$

where,

$W_{s}$ is the strain energy function,

$\mu$ and $\lambda$ are Lame elastic constants,

$J_{e l}$ is the elastic deformation gradient,

$I_{1}$ is the scalar invariant of $\mathrm{C}$.

TABLE V

COMPUTATION INFORMATION

\begin{tabular}{|c|c|}
\hline Computation time & $42 \mathrm{~s}$ \\
\hline $\mathrm{CPU}$ & $\begin{array}{r}\text { Intel(R) Core (TM) i3-3217U CPU @ } \\
1.80 \mathrm{GHz}, 2 \text { cores }\end{array}$ \\
\hline Operating system & Windows 8 \\
\hline
\end{tabular}

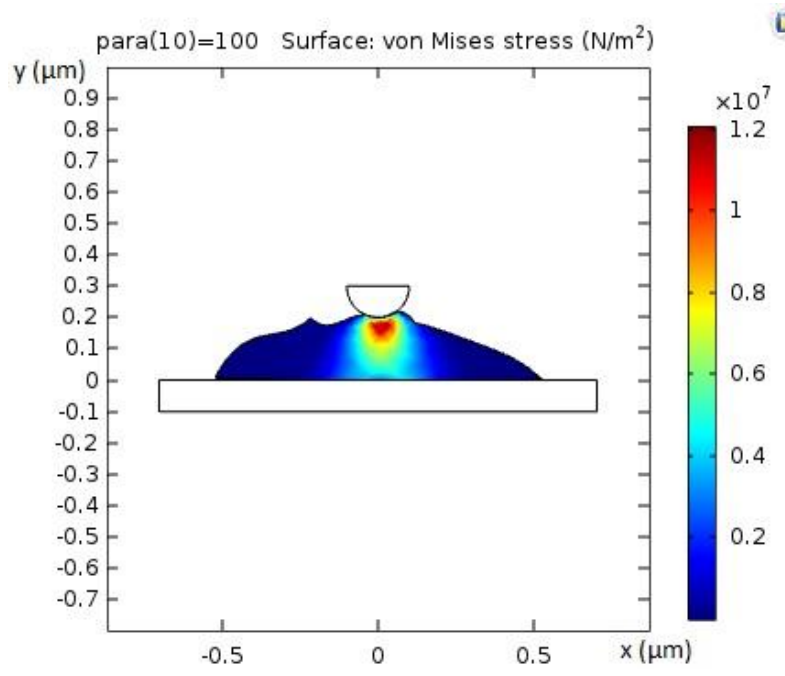

Figure 8: Von Mises stress in $\mathrm{N} / \mathrm{m}^{2}$

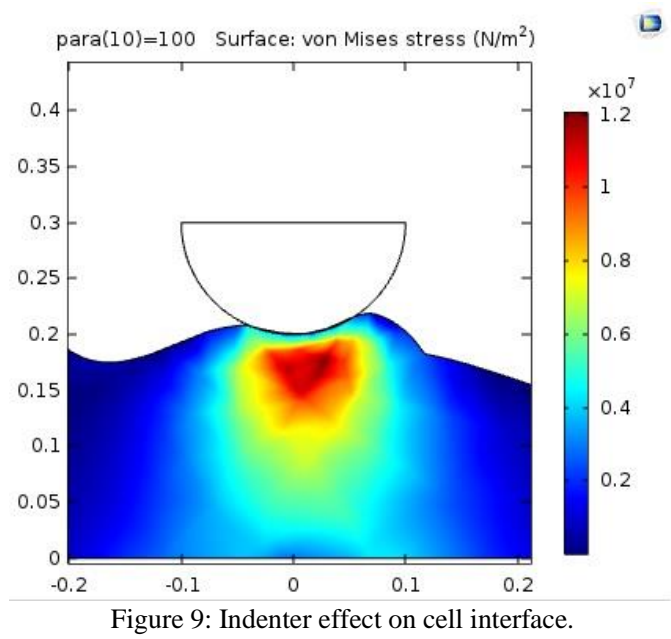

Materials: For cell material parameters we used data given in table 1. For rigid indentor and support which are in carbon-diamond, we used data given in table 2.

\section{B. Cell Model without Nucleus}

The numerical simulation was performed by the finite element method implemented in COMSOL multiphysics 5.2 software $[13,15,35]$.

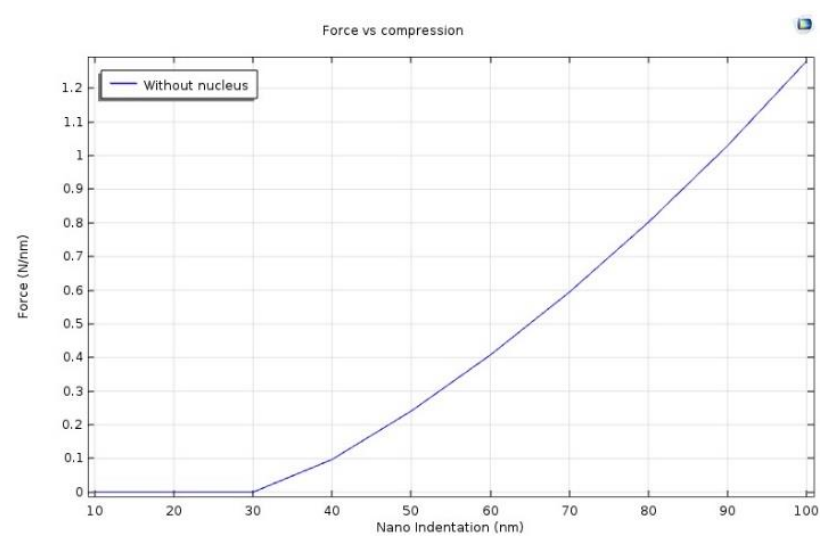

Figure 10: Force vs compression curve for cell without nucleus.
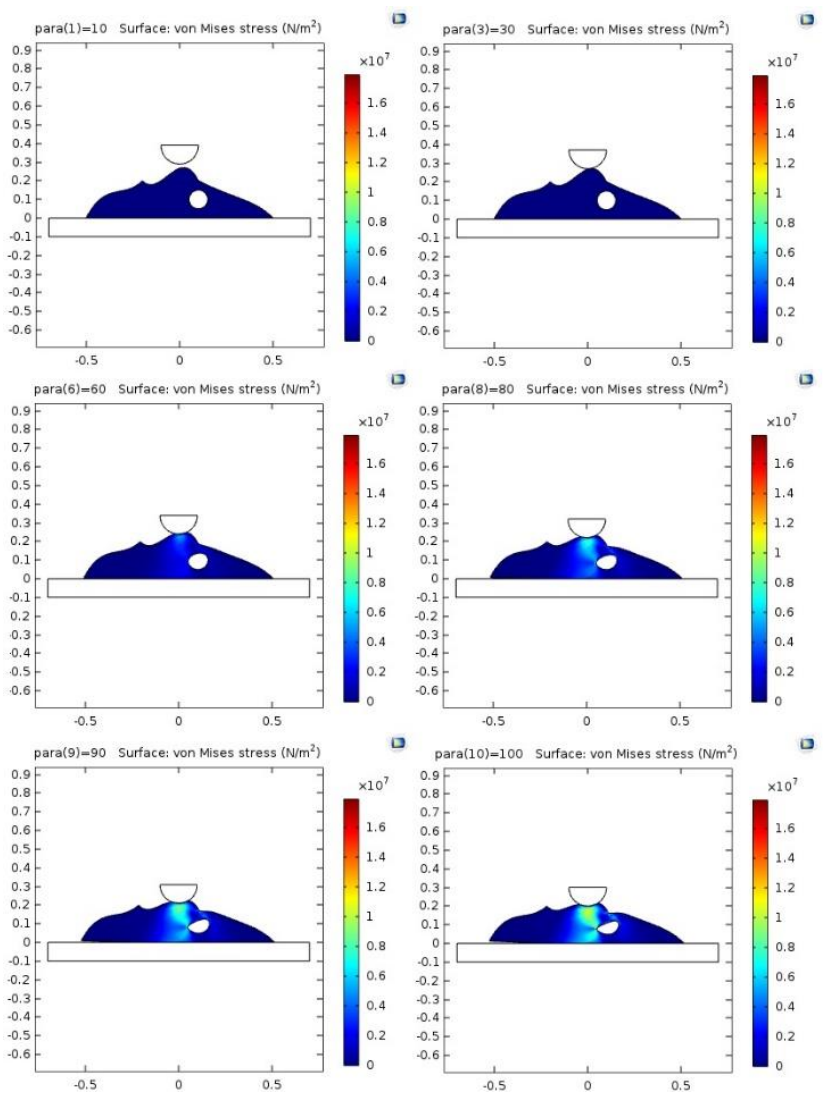

Figure 11: Von Mises indentation steps $\mathrm{N} / \mathrm{m}^{2}$.

Geometry: The 2D geometry model gives an approximation to the real cell with the assumption that cell out of plane thickness is equal to $10 \mathrm{~nm}$. In the plane strain 
approximation this setting only affects total force computations. The cell total length is chosen equal to $1000 \mathrm{~nm}$ and the height is equal to $255 \mathrm{~nm}$. The cell shape presents a tensegrity form $[1,3,6,7]$. The circular indenter diameter is equal to $200 \mathrm{~nm}$. The support is modeled at the bottom part of cell, as shown in figure 3.

Mesh Generation: The mesh generation was chosen to be normal triangular elements, and it was physics controlled which means that COMSOL refines mesh in the critical regions. The mesh detail obtained is given in the table 3 .

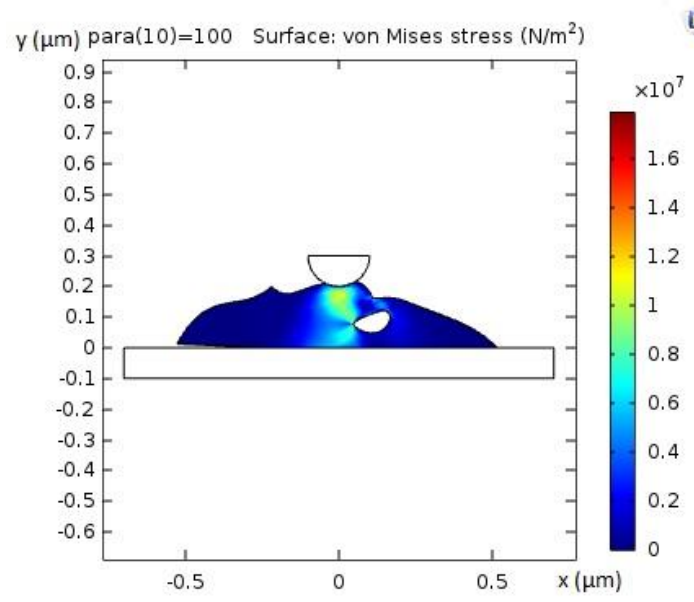

Figure 12: Von Mises stress in $\mathrm{N} / \mathrm{m}^{2}$.

TABLE VI

COMPUTATION INFORMATION

\begin{tabular}{|c|c|}
\hline Computation time & $50 \mathrm{~s}$ \\
\hline $\mathrm{CPU}$ & Intel(R) Core(TM) i3-3217U CPU @ \\
& $1.80 \mathrm{GHz}, 2$ cores \\
\hline Operating system & Windows 8 \\
\hline
\end{tabular}

Once the mesh obtained figure 4, we launch the computational operations which were described in section III. A. i.e. mechanics cell behavior without nucleus.

\section{Cell Model with Nucleus}

Geometry: For the cell model with nucleus geometry, we used the same data from model without nucleus, with the nucleus geometry adding. We choose the nucleus diameter equal to $100 \mathrm{~nm}$ as shown in figure 5 .

Mesh Generation: The mesh generation was chosen to be normal triangular elements, and it was physics controlled which means that COMSOL refines mesh in the critical regions. The mesh detail obtained is given in the table 4 .
Once the mesh obtained figure 6, we launch the computational operations described in section III. B. i.e. mechanics cell behavior with nucleus.

\section{RESULTS AND DISCUSSIONS}

The contact between the rigid base and cell is chosen to be prescribed displacement. We obtain results after the indenter vertical displacement along the y direction. We perform a displacement equal to $100 \mathrm{~nm}$ in which an indentation equal to $55 \mathrm{~nm}$. The CPU time is equal to $42 \mathrm{~s}$.

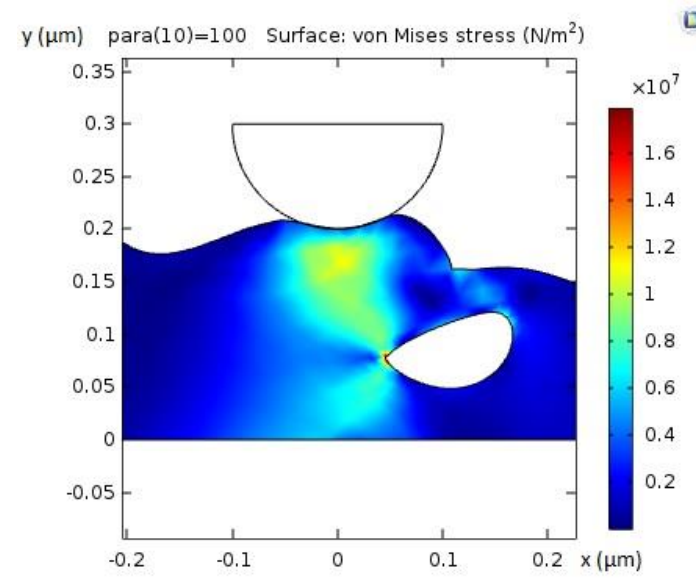

Figure 13: Indenter effect on cell interface.

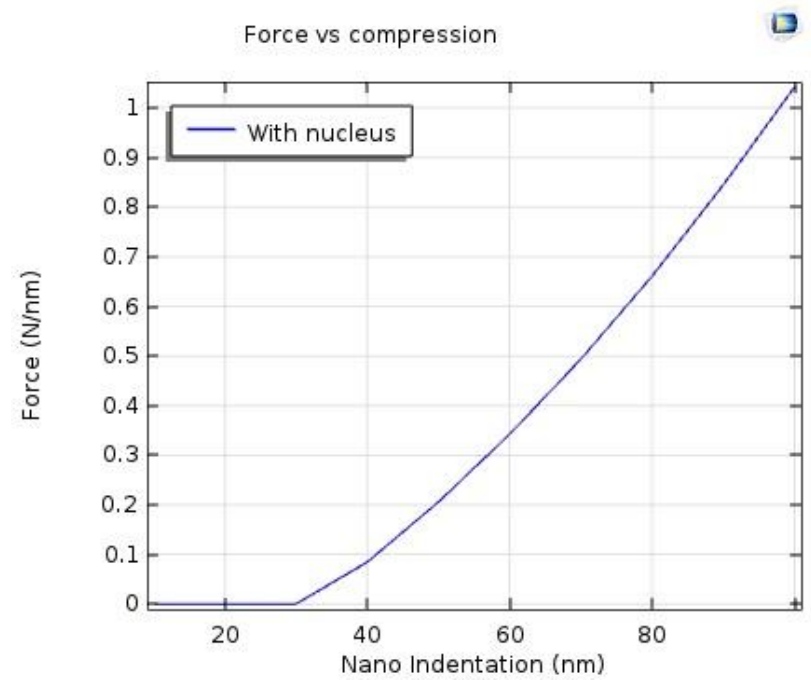

Figure 14: Force vs compression curve for cell with nucleus.

\section{A. Mechanics Cell Behavior without Nucleus}

For the cell without nucleus the obtained CPU time is equal to $42 \mathrm{~s}$, the computational data are given in table 5 .

The different nanoindentation steps following y axis are given in Fig. 7 in which the von Mises stress in $N / \mathrm{m}^{2}$ is the main result. We observe that the maximum stress is equal to 
$1.2 \times 10^{7} \mathrm{~N} / \mathrm{m} 2$ and is concentrated in the region below the indenter.

Figures 7, 8 and 9 shows the von mises stress repartition in the cell without nucleus compressed by a rigid carbon nanoindenter.

These results are in good agreements with those obtained with experiments $[5,22]$.

\section{B. Mechanics Cell Behavior with Nucleus}

For the cell with nucleus, the obtained CPU time is equal to $50 \mathrm{~s}$, the computational data are given in table 6 .

The different nanoindentation steps following $\mathrm{y}$ axis are given in figure 11 in which the von mises stress in $N / \mathrm{m}^{2}$ is the main result. We observe that the maximum stress is equal to $1.7 \times 10^{7} \mathrm{~N} / \mathrm{m}^{2}$ and is concentrated in the corner near compressed nucleus.

Figures 11, 12 and 13 shows the von Mises stress repartition in the cell with nucleus compressed by a rigid carbon nanoindenter.

These results in figure 10 and figure 14 are in good agreements with those obtained with experiments $[5,22]$.

\section{CONCLUSIONS}

This work shows the nanoindentation cell mechanical characteristics. We investigated the cases in which the main assumptions was first to chose a mathematical model for the rigid part and hyperelastic part, the second assumption was to chose the numerical method which can resolve the mathematical model and to give numerical results in adequation with theoretical and experimentals ones. The models used are cell without nucleus and cell with nucleus. The main result is the observation of a von Mises stress increse for the cell in which a nucleus is incorporated.

\section{REFERENCES}

[1] L. Chua, "Finite Element Modelling of Living Cells", Final Report of Mechanical Engineering Individual Project, The University of Edinburg, 2013/2014.

[2] Y. Ding, X. Niu, G. Wang, "Elastic compression of nanoparticles with surface energy", J. Phys. D: Appl. Phys. 48, 7pp, 2015.
[3] D. E. Ingber, "The architecture of life", Scientic. C American, Inc. 48-57, 1998.

[4] M. Zhang, Y. Cao, G. Li, X. Feng, "Spherical indentation method for determining the constitutive parameters of hyperelastic soft materials", Biomech Model Mechanobiol. 13:1-11, 2014.

[5] Michelle L. Oyen, Handbook of nanoindentation with biological applications. Pan Stanford Publishing Pte. Ltd. 2011.

[6] B. Fallqvist, "On the mechanics of actin and intermediate filament networks and their contribution to cellular mechanics" KTH school of engineering sciences, royal institute of technology, Stockholm, Sweden, 2015.

[7] D. E. Ingber, "Tensegrity I. Cell structure and hierarchical systems biology". Journal of Cell Science, 116, 1157-1173, 2003

[8] Comsol software licence agreement, "Fluid-structure interaction in a network of blood vessels".

[9] D. W. Pepper, J. C. Heinrich, The finite element method. CRC Press, Taylor \& Francis group, 2017.

[10] M. Sato et al., "Local mechanical properties measured by atomic force microscopy for cultured bovine endothelial cells exposed to shear stress". Journal of Biomechanics, 33, 127$135,2000$.

[11] E. A-Hassan et al., "Relative microelastic mapping of living cells by atomic force microscopy” Biophysical journal, 74, 1564-1578, 1998.

[12] L. Chen et al., "Modeling effect of surface roughness on nanoindentation tests" Procedia CIRP 8, 334-339, 2013.

[13] Comsol Multiphysics, "Essentials of postprocessing and visuaisation in COMSOL multiphysics ${ }^{\circledR}$ ”, Comsol Handbook series, 2015.

[14] R. Garcia, E. T. Herruzo, "The emergence of multifrequence force microscopy”, Nature nanotechnology, 7, 217-226, 2014.

[15] Th. Reuter, M. Hoffmann, "A elastic and hyperelastic material model of joint cartilage- Calculation of the pressure dependent material stress in joint cartilage", COMSOL conference, Stuttgart, 2011.

[16] Y. Ding et al., "Compression of hyperelastic cells at finite deformation with surface energy", International journal of applied mechanics, vol. 8, no. 6, 2015.

[17] Y. Ding et al., "On the determination of elastic moduli of cells by AFM based indentation", Nature Scientific reports, 7, 2017. 
[18] S. Dokos, "Modelling organs tissues, cells and devices using Matlab and Comsol multiphysics", Springer-verlag, Berlin, Heidelberg, 2017.

[19] G. Ferrazzi, "Numerical modeling of Atomic Force Microscopy towards estimation of material parameters from fibroblast cells”, Degree project in solid mechanics second level, Stockholm, Sweden, 2011.

[20] P. M. Schön, M. Gosa, G. J. Vancso, "Imaging of mechanical properties of soft matter from heterogeneous polymer surfaces to singles biomolecules", Imaging and microscopy, 2012.

[21] W. K. Liu et al., "Imersed finite element method and its applications to biological systems", Comput. Methods Appl. Mech. Engrg. 195, 1722-1749, 2006.

[22] M. Plodinec "Probing the determinants of cellular elasticity by AFM", Thesis, Basel, 2012.

[23] J. Marra, J. Israelachvili "Direct measurements of forces between phosphatidylcholine and phosphatidylethanolamine bilayers in aqueous electrolyte solutions ", Biochemistry, 24, 4608-4618, 1985.

[24] H. Ladjal et al. "Micro to nano biomechanical modeling for assisted biological cell injection”, Hal archives ouvertes, 2013.

[25] S. A. James et al., "Atomic force microscopy of biofilmsimaging, interactions, and mechanics”, Intech open science, chapter 6, 95-118, 2016.

[26] Y. M. Efremov et al., “Anisotropy vs isotropy in living cell indentation with AFM", Nature Scientific reports, 9:5757.
[27] A. Boccaccio et al., "Nanoindentation characterization of human colorectal cancer cells considering cell geometry, surface roughness and hyperelastic constitutive behaviour", IOP Publishing Ltd. Nanotechnology 28, 2017.

[28] Q. Yang et al., "Nanoindentation experiment and modeling for biomechanical behavior of red blood cell", Proceeding of the IEEE, 2011.

[29] G. Tang et al., "Biomechanical heterogeneity of living cells: comparison between atomic force microscopy and finite element simulation”, Langmuir 35, 7578-7587, 2019.

[30] M. Marsal et al., "AFM and microrhelogy in the zebrafish embryo yolk cell", Journal of visualized experiments 129 , 2017.

[31] O. Sahin et al., "An atomic force microscope tip designed to measure time-varying nanomechanical forces", Nature Nanotechnology 2, 2007.

[32] K. K. M. Sweers et al., "Spatially resolved frequencydependent elasticity measured with pulsed force microscopy and nanoindentation”, Nanoscale 4, 2072-2077, 2007.

[33] M. Radmacher et al., "Imaging adhesion forces and elasticity of lysozyme adsorbed on mica with the atomic force microscope”, Langmuir 10, 3809-3814, 1994.

[34] F. Rico et al., "Mechanical mapping of single membrane proteins at submolecular resolution”, Nano letters, 39833986, 1994.

[35] https://www.comsol.com/blogs/fitting-measured-datato-different-hyperelastic-material-models/ 